\title{
Investigación en Progreso: Tecnologías Informáticas de Mediación de Interacción. Aplicación a la formación de Recursos Humanos
}

\author{
Pablo Augusto Sznajdleder ${ }^{1,2}$ \\ 1. Programa de Maestría en Ingeniería de Sistemas de Información. \\ Escuela de Posgrado, Facultad Regional de Buenos Aires. Universidad Tecnológica Nacional. Argentina. \\ 2. Laboratorio de Investigación y Desarrollo de Espacios Virtuales de Trabajo. \\ Grupo de investigación en Sistemas de Información. Universidad Nacional de Lanús. Argentina. \\ pablosz@pablosz.com.ar
}

\begin{abstract}
Resumen-El presente documento describe el proyecto de investigación, cuyo objetivo es desarrollar una metodología de enseñanza basada en las tecnologías de mediación de interacción disponibles, para ser aplicado tanto en el ámbito académico cómo en el profesional.
\end{abstract}

Palabras Clave-Capacitación, Educación, YouTube, Facebook, Redes Sociales.

\section{JUSTIFICACIÓN}

En [1] se sostiene que el advenimiento de la Web 2.0 ha transformado el paisaje de Internet; que pasó de ser un "depósito" de datos multimedia estático a convertirse en un "hábitat" dinámico y participativo de las personas. Los videos en la web, mediados en ambientes como YouTube, son adecuados para el diseño de aprendizajes centrados en el estudiante; donde éstepuede seleccionar qué videos desea utilizar para mejorar su apropiación de saberes.

Ketterl y Morisse [2] señalan que la grabación de las clases como recurso didáctico ha sido utilizado durante varios años; con diferentesmodos de distribución. Los autores citados desarrollaron un enfoque de aprendizaje mixto basado en la grabación de conferencias en el cual dichas grabaciones sustituyen a las clases presenciales.

El video web creado por el docente puede ser compartido, libre y abiertamente, por cualquier persona a través de Internet; y su valor radica en el contenido y la forma que el autor eligió para comunicar. [1].

Atmojoy y Bandung [3] sostienen que los contenidos enseñados medianteel uso de videos por Internet suelen ser más fácilmente comprendidos por los estudiantes que aquellos contenidos que son transmitidos a través de los mecanismos tradicionales.

York y Owston [4] exploran las oportunidades y desafíos de la integración de videos que, publicados en Internet $\mathrm{y}$ puestos a disposición de los estudiantes, constituyen un nuevo modelo de enseñanza para la educación universitaria; diseñado para facilitar lo que se conoce como "la capacidad de aprendizaje del siglo 21" [5].

Los autores precitados analizan las cualidades del video web generado por el docente y ofrecen un marco conceptual para un modelo de instrucción de aprendizaje mediado por este tipo de recursos; y proporcionan evidencia empírica que apoya la efectividad del modelo y mide el impacto de su aplicación en el aprendizaje de los alumnos universitarios.

\section{FUNDAMENTOS}

Bravo Ramos [6], señala que un video educativo es aquél que cumple un objetivo didáctico previamente formulado. Sostiene además que es importante que, para que los alumnos valoren la información que les suministra el video, puedan identificar el contenido del mismo con el programa de la asignatura; de forma tal que le otorguen la categoría de texto oficial. Si además advierten que los profesores han participado en la realización del vídeo su apreciación e interés será mucho mayor.

Lewis [7], sostiene que los materiales didácticos universitarios tienen que formar verdaderos paquetes didácticos integrados poraudio, vídeo, diapositivas, textos y software. Estos materiales deben ser diseñados para un doble uso: tanto los estudiantes presenciales, como aquellos que no pueden estar físicamente presentes, conseguirán elacceso al aprendizaje a través de una variedad de medios y con la posibilidad de clases tutoriales y entrevistas personales.

Cabero y Llorente [8] comparan la ponderación que varios autores le asignan a diversos atributos de las TIC (Tecnologías de Información y Comunicación) poniendo en evidencia que, aunque existen diferentes puntos de vista sobre la contribución que las TIC aportan a la educación, la gran mayoría coincide en destacar que su aplicación en el ámbito educativo favorece y potencia la motivación del alumno.

Además, Cabero y Llorente le atribuyen a las TIC el poder de favorecer la ruptura de las variables espacio/temporales en las que tiende a desenvolverse el profesor y el estudiante, establecer posibilidades de comunicación tanto sincrónica como asincrónica, y favorecer la interacción profesor/estudiante y estudiante/estudiante. Abordajes similares son presentados en Khan [9].

\section{OBJETIVO}

Se pretende identificar las tecnologías de mediación de interacción aplicables a la formación de recursos humanos y definir un método que identifique la articulación del uso de estas tecnologías en los procesos formativos. Se buscará la validación del método propuesto mediante trabajo experimental en cursos universitarios. Se espera demostrar en la investigación que las tecnologías informáticas de mediación identificadas, al ser utilizadas de la manera definida en el método propuesto, permiten obtener un mejor rendimiento del proceso formativo. 


\section{Metodología de Desarrollo}

Para construir el conocimiento de la presente investigación, se seguirá un enfoque de investigación clásico [10-11] con énfasis en la producción de tecnologías [12]; identificando los métodos y materiales necesarios para desarrollar el proyecto.

\section{A. Métodos}

A continuación se definen los métodos que se llevarán a cabo en el presente trabajo:

\section{1) Revisiones Sistemáticas}

Las revisiones sistemáticas [13] de artículos científicos siguen un método explícito para resumir la información sobre determinado tema o problema; se diferencia de las revisiones narrativas en que provienen de una pregunta estructurada y de un protocolo previamente realizado.

\section{2) Prototipado evolutivo experimental, método de la ingeniería}

El prototipado evolutivo experimental [14] consiste en desarrollar una solución inicial para un determinado problema, generando su refinamiento de manera evolutiva por prueba de aplicación de dicha solución a casos de estudio (problemáticas) de complejidad creciente. El proceso de refinamiento concluye al estabilizarse elprototipo en evolución.

\section{B. Materiales}

En este investigación se utilizaran los siguientes materiales:

- Formalismos de ingeniería de software orientados al modelado y diseño de interfases.

- Servidores de video-streaming y redes sociales.

\section{Metodología}

En esta investigación se seguirán los siguientes pasos:

- Realizar una investigación documental exploratoria sobre modelos de proceso aplicables al desarrollo, selección y uso de tecnologias informaticas de mediación de interacción.

- Identificar casos de estudio y casos de validación en el area de formación de recursos humanos mediado por tecnologías informáticas.

- Desarrollar mediante la metodología de prototipado evolutivo un modelo de proceso de desarrollo, de selección y de uso de las tecnologias informaticas de mediación de interacción que identifique fases, tareas, técnicas de representación y procedimientos de ejecución de cada tarea.

- Realizar pruebas de concepto en los casos de estudio y casos de validación identificados buscando corroborar el modelo de proceso propuesto.

\section{REFERENCIAS}

[1] Lupshenyuk, D., Hocutt, M., Owston, R. 2011, Web Video Project As An Instructional Strategy In Teacher Education. Society For Information Technology \& Teacher Education International Conference. Vol. 2011. No. 1. Pág 984-991.

[2] Ketterl, M., Morisse, K. 2009, User Generated Web Lecture Snippets To Support A Blended Learning Approach. Proceedings Of 2009 World Conference On Educational Multimedia, Hypermedia And Telecommunications. Pág. 28862893

[3] Atmojo, K., Bandung, Y. 2012, Web Video To Support Digital Learning In Rural Primary Schools. IEEE International Conference On Cloud Computing And Social Networking (ICCCSN). Pág. 1-4.

[4] York, D., Owston, R. 2012. Enabling Learning With UserCreated Web Video In Higher Education. Task Report. York
University. Canada. Http://Www.Yorku.Ca/Rowston/AERA 2012Dennis.Pdf. Vigente 21/04/2014.

[5] Thomas, D., Brown, J.2009. The Play of Imagination: Extending The Literary Mind. En After Cognitivism. Pág. 99120. Springer.

[6] Bravo Ramos, L. 1996, ¿Qué es el vídeo educativo?. Disponible en http://rabida.uhu.es/dspace/bitstream/handle/ 10272/766/b153 46043.pdf?sequence=1, página vigente al: 14 de noviembre de 2014.

[7] Lewis, R. 1998, Staff development in conventional institutions moving towards open learning. Disponible en: http://www. c31.uni-oldenburg.de/cde/support/readings/lewis98.pdf, página vigente al día 5 de marzo de 2015.

[8] Cabero, J. y Llorente, Ma ${ }^{\text {a }}$ 2005, Las TIC y la Educación Ambiental. Revista Latinoamericana de Tecnología Educativa, 4 (2), 9-26. Disponible en: http://mascvuex.unex.es/revistas /index.php /relatec/ article/view/197, página vigente al día 14 de noviembre de 2014

[9] Kahan, S. Salman Khan talk at TED 2011. 2011, https://www. khanacademy.org/talks-and-interviews/conversations-with-sal/ $\mathrm{v} /$ salman-khan-talk-at-ted-2011-from-ted-com.

[10] Riveros, H. y Rosas, L. 1985. El Método Científico Aplicado a las Ciencias Experimentales. Editorial Trillas. ISBN 96-8243893-4.

[11] Creswell, J. W. (2002). Educational research: Planning, conducting, and evaluating quantitative. Prentice Hall.

[12] Sabato J, Mackenzie M. 1982. La Producción de Tecnología: Autónoma o Transnacional. Instituto Latinoamericano de Estudios Transnacionales - Technology \& Engineering. ISBN 9789684293489.

[13] Argimón J. 2004. Métodos de Investigación Clínica y Epidemiológica. Elsevier España, S.A. ISBN 9788481747096.

[14] Basili, V. 1993. The Experimental Paradigm in Software Engineering. En Experimental Software Engineering Issues: Critical Assessment and Future Directions (Ed. Rombach, H., Basili, V., Selby, R.). Lecture Notes in Computer Science, Vol. 706. ISBN 978-3-540-57092-9

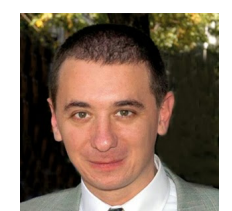

Pablo Augusto Sznajdleder. Ingeniero en Sistemas de Información egresado de la Universidad Tecnológica Nacional (UTN.BA, 1999). Es profesor ordinario con categoría de asociado en la cátedra de "Algoritmos y estructura de datos" $\mathrm{y}$, promotor y profesor en la materia optativa: "Algoritmos avanzados para estructuras complejas"; ambas materias en UTN.BA. Es autor de los libros "JEE a fondo" (Alfaomega, 2015), "Algoritmos a fondo" (Alfaomega 2013), "Java a fondo" (Alfaomega, 2010/12) y "HolaMundo.pascal" (CEIT, 2007). Se desempeñó como instructor Java para Sun Microsystems y Oracle obteniendo, en 1997, las certificaciones SCJP y SCJD, que fueron las primeras certificaciones Java acreditadas en Argentina y estuvieron entre las primeras logradas en latinoamérica. Actualmente, se desempeña en el ámbito profesional como consultor e instructor en tecnologías Java/JEE proveyendo servicios de coaching y capacitación en las empresas líderes del país. Es candidato del Programa de Maestría en Ingeniería de Sistemas de Información de la Universidad Tecnológica Nacional e Investigador Tesista del Laboratorio de Investigación, Desarrollo e Innovación en Espacios Virtuales de Trabajo de la Universidad Nacional de Lanús. 